\title{
The Role of Splenectomy with Vascular Disconnection in Pediatric Patients with Hypersplenism, Portal Hypertension and Liver Disease
}

\author{
Pierre Jean Aurelus ${ }^{1 *}$, Hermilo De La Cruz Yáñez ${ }^{1}$, Edwin Allen Mejía Solís ${ }^{2}$, Elvia Ximena \\ Lemus Manjarrez ${ }^{3}$, María Antonieta Julián Núñez ${ }^{3}$, Rafael Roberto Zapata Carrión ${ }^{1}$ \\ ${ }^{1}$ Department of Gastro-transplantation, Pediatric Hospital of CMNSXXI (IMSS), México. \\ ${ }^{2}$ Department of General Surgery, Regional Hospital of ISSSTE, Puebla, Mexico. \\ ${ }^{3}$ Department of Pediatric Surgery, Pediatric Hospital of CMNSXXI (IMSS), México. \\ aurelusjean@yahoo.com.mx
}

\begin{abstract}
Introduction: Portal hypertension and its complications are the main cause of morbidity and mortality in pediatric Patients with cirrhosis or end-stage liver disease. Those Patients should require a threshold value of hepatic venous pressure gradient of 10 to $12 \mathrm{mmHg}$ for the development of varices. The aim of this work was to evaluate the influence of splenectomy with disconnection of short veins of stomach by using Child-TurcottePugh score in a small case series of four Pediatric Patients with liver disease or cirrhosis.

Patients: From July 2016 to august 2017, 4 patients with liver disease were diagnosed with hypersplenism or splenomegaly and portal hypertensive gastropathy. Underwent laparoscopic or open procedure, we performed splenectomy with disconnection of azygos or stomach short veins (vascular disconnection).

Outcomes: The patients 1,2 and 4 had a good outcome, the platelet account was recovered and no ascites and encephalopathy. Prothrombin time was perfectly and they not required variceal endoscopic treatment at month after the procedure. Endoscopic valuation showed variceal gastropathy hypertensive mild, that not required endoscopic therapy and the medical therapies with non-selective Beta-blocker were decreased; the Child-Pugh class B was changed to class A. Patient 3: presented the same outcome, even though the Child-Pugh class A was not altered.

Conclusion: The splenectomized pediatric patient requires careful monitoring in the early postoperative period to identify some complications. Fortunately, in this study we observed a good outcome, amelioration of the portal hypertension gastropathy and a better function of liver.
\end{abstract}

Key Words: Portal hypertension- Liver function- Splenectomy- Vascular disconnection-Variceal bleeding

\section{INTRODUCTION}

Portal hypertension (PH) and its complications are the main cause of morbidity and mortality in pediatric Patients with cirrhosis or end-stage liver disease. ${ }^{1}$ in fact, hypersplenism or splenomegaly like $\mathrm{PH}$ complication is considered a risk of leucopenia, bleeding, variceal formation, variceal bleeding and capsular disruption. However, the triad of splenomegaly, leucopenia and liver cirrhosis, is known as Banti's disease. ${ }^{1-3}$ The initial event that leads to the development of portal hypertension is the increased intrahepatic resistance that is due to architectural disturbances and sinusoidal contractile elements. ${ }^{2}$ Patients with cirrhosis and sinusoidal portal hypertension should required a threshold value of hepatic venous pressure gradient of 10 to $12 \mathrm{mmHg}$ for the development of varices. ${ }^{1,2}$ The mortality rate from variceal bleeding in cirrhotic patients with portal vein system thrombosis is 30\%-70\%. ${ }^{1}$ Finally, Portal hypertension in patients with liver cirrhosis is characterized primarily by increased intrahepatic vascular resistance and increased splanchnic blood flow. ${ }^{2,3}$ 
The Role of Splenectomy with Vascular Disconnection in Pediatric Patients with Hypersplenism, Portal Hypertension and Liver Disease

Cirrhotic patients with portal hypertension have different levels of hypersplenism. ${ }^{3,4}$ The decrease of hepatic blood flow further damaged to the liver function, even lead to liver failure, hepatic encephalopathy and even death. Under an adequate conditions, near of $30 \%$ of the whole body blood platelets stored in the spleen, but it can increase to $80 \%$ in view of the hypersplenism, splenomegaly. ${ }^{1-4}$ Therefore, splenectomy has been an option indicated to patients who suffer from hypersplenism with chronic liver diseases. Furthermore, several reports described a decrease in the portal inflow and pressure after splenenctomy. ${ }^{4-6}$ The aim of this work was to evaluate the influence of splenectomy with disconnection of azygos or short veins of stomach (vascular disconnection) by using Child-Turcotte-Pugh score and classification in a small series of four Pediatric Patients with liver disease or cirrhosis, portal hypertension and hypersplenism in our Medical Center (Pediatric Hospital "Dr. Silvestre Frenk Freund", National Medical Center Siglo XXI/ México).

\section{PATients}

From July 2016 to august 2017, 4 patients with liver disease were diagnosed with hypersplenism or splenomegaly and portal hypertensive gastropathy due to portal hypertension in our gastro-transplantation department required treatments. Underwent laparoscopic or open procedure, we performed splenectomy with disconnection of azygos or stomach short veins (vascular disconnection). The inclusion criteria were as follows: clinical bleeding history, splenomegaly secondary to hypersplenism and thrombocytopenia (platelets account (PLT) $<40 \mathrm{X} 10^{4} / \mathrm{L}$ ), Child-Pugh liver function. Retrospectively collected preoperative data of the patients, age, gender, and weight, etiology of cirrhosis, ascites, encephalopathy, platelet count (PLT), prothrombin time (PT), international normalized ratio (INR), encephalopathy, albumin, serum bilirubin levels (TBIL), alanine transaminase (ALT), glutamic oxaloacetic transaminase (AST). The four patients were received Pneumovax Vaccine from 15 to 20 days before the procedure with intravenous antibiotics as a preoperative prophylaxis. Endoscopy procedure, computed abdominal tomography and Doppler ultrasound were evaluated.

\section{CASE 1}

A 3-year-old male of $15 \mathrm{~kg}$ of weight and $1.2 \mathrm{~cm}$ of size, was referred to our center with 2 years' history of hypersplenism secondary to portal hypertension and portal cavernous transformation (PCT). During this time, he had prior history of oesophagic variceal and portal hypertensive gastropathy. Physical examination showed evidence of hepatosplenomegaly and Slight ascites. The patient had a good answer with the treatments for PH but he has continued with thrombocytopenia. He had TBIL $1.8 \mathrm{mg} / \mathrm{dl}$; INR 1.7, PT of 17 seconds, albumin serum of $3.3 \mathrm{~g} / \mathrm{dl}$, ALT $200 \mathrm{UI} / \mathrm{L}$, AST $416 \mathrm{UI} / \mathrm{L}$, PLT $38 \times 10^{4}$ and she had Child-Pugh class B. Abdominal ultrasound and computed tomography angiography revealed portal cavernous, permeability of the spleen vasculature and the larger of the spleen (figures: 1,2). The patient was evaluated and we performed a laparoscopic splenectomy with ligation of short stomach veins.

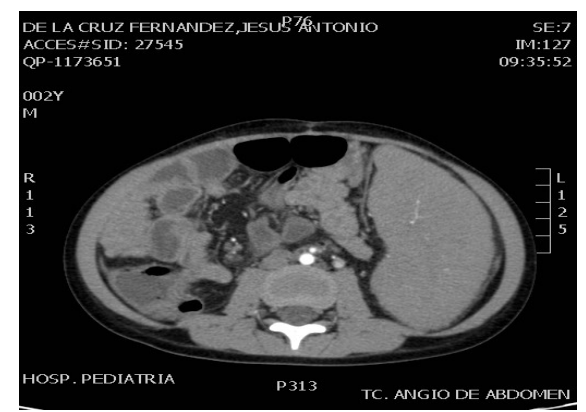

Fig1. Computed abdominal tomography angiography.

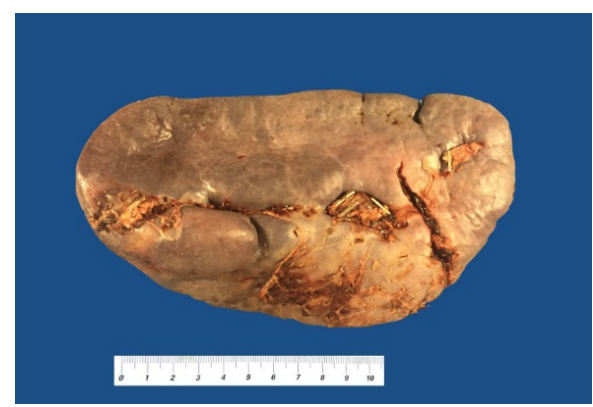

Fig2. spleen size 
The Role of Splenectomy with Vascular Disconnection in Pediatric Patients with Hypersplenism, Portal Hypertension and Liver Disease

\section{CASE 2}

A 9-year-old male of $25.6 \mathrm{~kg}$ of weight and $1.32 \mathrm{~cm}$ of size, with multiples surgical procedures secondary gastroesophagic reflux, hemangioma and portal hypertension, he was referred to our department with 5 years' history of hypersplenism, portal hypertensive gastropathy (PHG), PCT and cirrhosis. The child had history of variceal bleeding due to severely gastro esophageal varices requiring ligation variceal endoscopic and medical therapy with non-selective beta-blockers. Physical examination showed evidence of hepatosplenomegaly, moderate ascites and abdominal distension. Preoperative laboratory tests showed serum bilirubin $1.26 \mathrm{mg} /$ $\mathrm{dl}$ and INR 1.34; TP of 13.4 seconds, albumin serum of $4.5 \mathrm{~g} / \mathrm{dl}$ and grade 2 of encephalopathy. However, with the medical therapy, the patient continued with thrombocytopenia with platelet count of $33 \mathrm{X} 10^{4}$ and recurrent variceal bleeding. He had Child-Pugh class B and severe varices in the last endoscopic before the procedure. Abdominal ultrasound and computed tomography angiography (CTA) revealed cirrhosis, PCT, permeability of the spleen vasculature and the larger of the spleen (figures: 3,4 ). The patient was evaluated and we performed an open splenectomy with ligation of short stomach veins and liver biopsy.

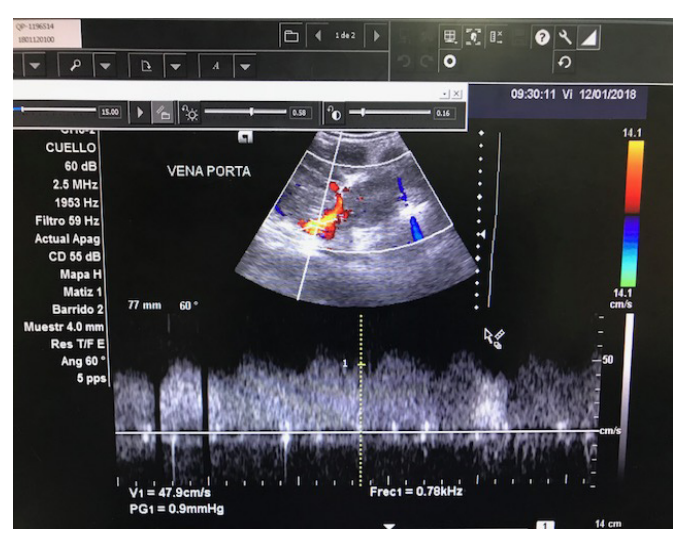

Fig3. Doppler of portal vein

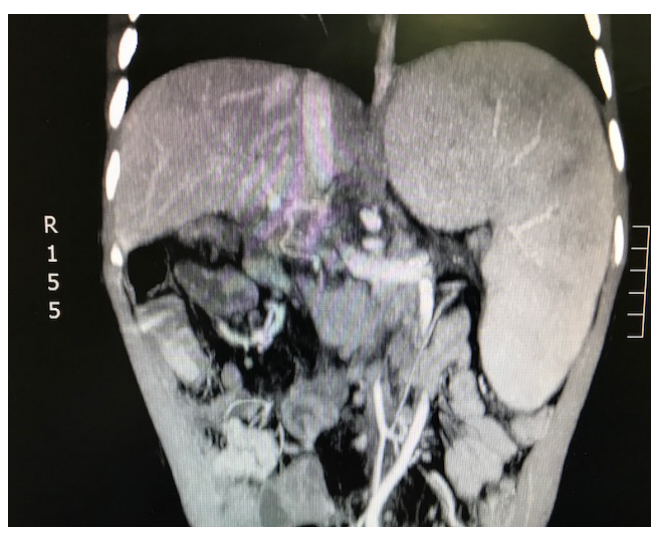

Fig4. CTA showed PCT and spleen size

\section{CASE 3}

A 5-month-old female of $3.5 \mathrm{~kg}$ of weight and $0.56 \mathrm{~cm}$ of size, with progressive familial intrahepatic cholestasis type 2 (PFIC2); she was referred to our department with hypersplenism secondary to portal hypertension and splenomegaly. Her Physical examination showed evidence of hepatosplenomegaly and Slight ascites. She had TBIL of $18 \mathrm{mg} / \mathrm{dl}$ and INR 1.7; PT of 17 seconds, albumin serum of $3.3 \mathrm{~g} / \mathrm{dl}$, ALT of $200 \mathrm{UI} / \mathrm{L}$, AST of $416 \mathrm{UI} / \mathrm{L}$, platelets account (PLT) 6 X $10^{4}$ and she had Child-Pugh class A.

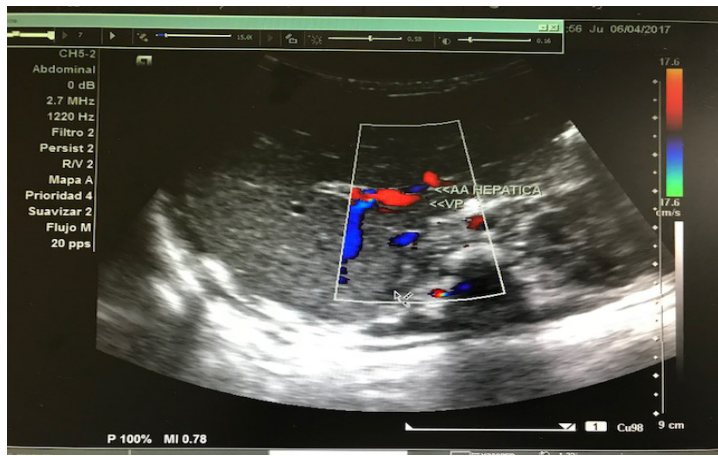

Fig5. Portal Doppler ultrasound

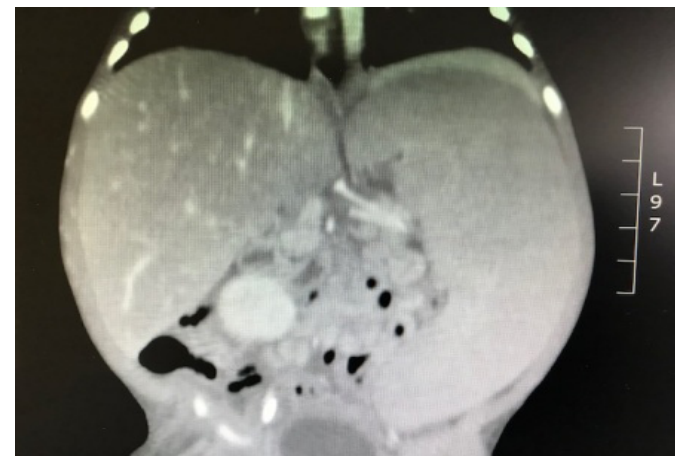

Fig6. CTA showed spleen size. 
The Role of Splenectomy with Vascular Disconnection in Pediatric Patients with Hypersplenism, Portal Hypertension and Liver Disease

Underwent the medical treatments for the $\mathrm{PH}$, she continued with thrombocytopenia with platelet count below $40 \times 10^{4}$. Abdominal ultrasound and computed tomography revealed liver disease; permeability of the spleen vasculature and the larger of the spleen (figures: 5, 6). She was evaluated and we performed a laparoscopic splenectomy (LS) with ligation of short stomach veins (LSSV) and liver biopsy.

\section{CASE 4}

A 9.6- year-old female of $30 \mathrm{~kg}$ of weight and $1.3 \mathrm{~cm}$ of size, with cholestasis and cirrhosis secondary to biliary atresia was referred to our department. She had history of Kasai procedure and multiples endoscopic therapy for portal hypertensive variceal bleeding. Her Physical examination showed evidence of splenomegaly and hypersplenism secondary to portal hypertension and Slight ascites. She had serum bilirubin total of $0.49 \mathrm{mg} /$ $\mathrm{dl}$, INR 1.2, TP of 11 seconds, albumin serum of $3.9 \mathrm{~g} / \mathrm{dl}$, grade 2 of encephalopathy and she had Child-Pugh class B. Platelets account $10 \times 10^{4}$, AST 69 and ALT 74 UI/L. Furthermore, the medical treatments for the PH she continued with thrombocytopenia with platelet count below $40 \mathrm{X}_{10} 0^{4}$. Abdominal ultrasound and computed tomography revealed cirrhosis, permeability of the spleen vasculature and the larger of the spleen (figures: $7,8)$. The patient was evaluated and we performed an open splenectomy with ligation of short stomach and azygos veins and liver biopsy.

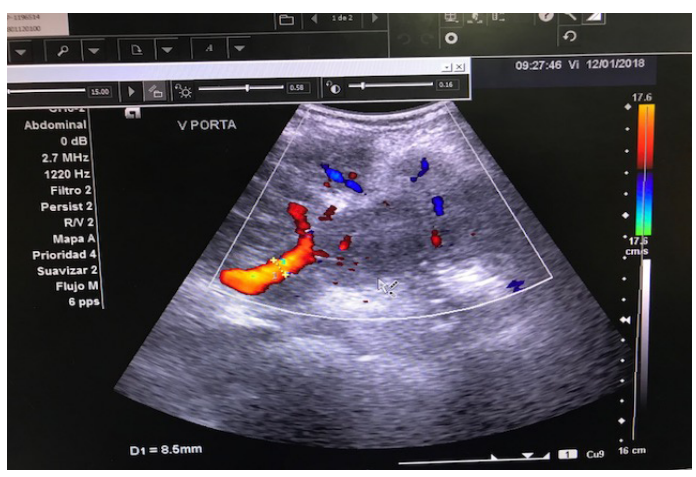

Fig7. Portal vein Doppler Ultrasound

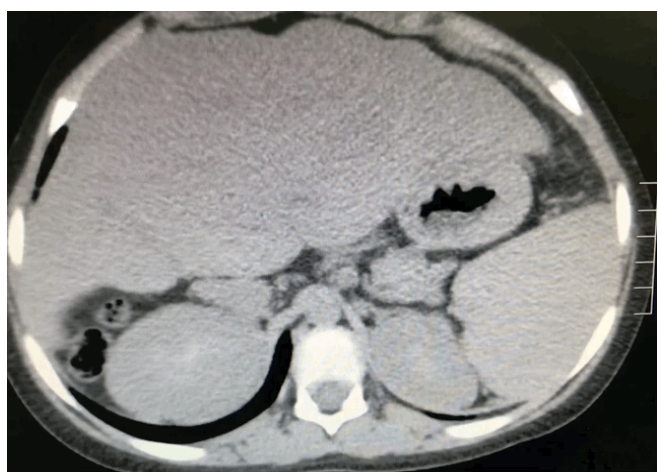

Fig8. CTA: cirrhotic liver /spleen size

\section{POSTOPERATIVE OUTCOMES}

The patient 1 had a good outcome and not required transfusion after the procedure, the platelets account were recovered, no ascites and encephalopathy, prothrombin time was normalized, international normalized ratio (INR) was 1.4 and serum bilirubin levels were $<1$ and he not required variceal endoscopic treatment at month after the procedure. The Child -Pugh class passed to A. Six months after the surgical procedure open splenectomy with disconnection of short gastric and azygoportal veins; patient 2 presented laboratory tests ammonium serum 48 ( range 11 - 48) with previous of 78, platelet account was $441 \times 10^{4}$, without ascites and encephalopathy; prothrombin time was 9.8 ( range 9.3 - 12.3), INR 0.98, total bilirubin serum $0.54 \mathrm{mg}$ / dl. Endoscopic valuation showed variceal gastropathy hypertensive mild, that not required endoscopic therapy and the medical therapy with non-selective Beta-blocker was decreased.

\begin{tabular}{|c|c|c|c|c|}
\hline Clinical Characteristics & Patient 1 & Patient 2 & Patient 3 & Patient 4 \\
\hline Etiology & $\begin{array}{l}-\mathrm{PH} \\
-\mathrm{PCT} \\
-\mathrm{HS}\end{array}$ & $\begin{array}{l}\text {-PH } \\
\text {-PCT } \\
\text {-HS, } \\
\text {-Cirrhosis } \\
\text {-PHG }\end{array}$ & $\begin{array}{l}\text {-HS } \\
\text {-Sickle cell disease }\end{array}$ & $\begin{array}{l}\text {-HS } \\
\text {-PH } \\
\text {-Cirrhosis } \\
\text { - BA }\end{array}$ \\
\hline
\end{tabular}


The Role of Splenectomy with Vascular Disconnection in Pediatric Patients with Hypersplenism, Portal Hypertension and Liver Disease

\begin{tabular}{|c|c|c|c|c|}
\hline $\begin{array}{l}\text {-Child-Pugh Class } \\
\text {-Platelets: } \\
\text {-AST(TGO): UI/L } \\
\text {-ALT( TGP): UI/L } \\
\text {-INR } \\
\text {-PHG } \\
\text {-Beta-blockers doses }\end{array}$ & $\begin{array}{l}\text { B } \\
38 \times 10^{4} \\
416 \\
200 \\
1.7 \\
\text { Moderate } \\
\text { High }\end{array}$ & $\begin{array}{l}\mathrm{B} \\
33 \times 10^{4} \\
37 \\
25 \\
1.34 \\
\text { Severe } \\
\text { High }\end{array}$ & $\begin{array}{l}\text { A } \\
6 \times 10^{4} \\
417 \\
200 \\
1.7 \\
\text { Moderate } \\
\text { High }\end{array}$ & $\begin{array}{l}\text { B } \\
10 \times 10^{4} \\
69 \\
74 \\
1.2 \\
\text { Severe } \\
\text { High }\end{array}$ \\
\hline Surgical Procedure & LS/LSSV & OS/LSSV & LS/LSSV & OS/LSSV/LAV \\
\hline \multicolumn{5}{|l|}{ Outcomes } \\
\hline $\begin{array}{l}\text {-Child-Pugh class } \\
\text {-Platelets: } \\
\text {-AST(TGO): UI/L } \\
\text {-ALT( TGP): UI/L } \\
\text {-INR } \\
\text {-PHG } \\
\text {-Beta-blockers doses }\end{array}$ & $\begin{array}{l}\text { A } \\
195 \times 10^{4} \\
71 \\
38 \\
1.4 \\
\text { Mild } \\
\text { Decreased }\end{array}$ & $\begin{array}{l}\text { A } \\
441 \times 10^{4} \\
45 \\
39 \\
0.98 \\
\text { Mild } \\
\text { Decreased }\end{array}$ & $\begin{array}{l}\text { A } \\
492 \times 10^{4} \\
390 \\
209 \\
1.2 \\
\text { Mild } \\
\text { Decreased }\end{array}$ & $\begin{array}{l}\text { A } \\
270 \times 10^{4} \\
59 \\
56 \\
0.94 \\
\text { Moderate } \\
\text { Decreased }\end{array}$ \\
\hline
\end{tabular}

PH: portal hypertension, PCT: portal cavernous transformation, HS: hypersplenism, PHG: portal hypertensive gastropathy, PC: progressive cholestasis, BA: biliary atresia. LAV: ligation of azygos vein.

The Child-Pugh class B was passed to class A. Patient 3: presented a good outcome. Platelet account was $250 \mathrm{x}$ $10^{4}$, prothrombin time was 11 (range 9.3-12.3), INR 1.2, total bilirubin serum $10 \mathrm{mg} / \mathrm{dl}$. The Child-Pugh class A was the same. AST was 390 and ALT was 209 UI/L. She had a mild portal hypertension without variceal bleeding during the time of follow-up. Patient number 4, six months after the procedure had serum ammonium 73 (range 11-48), platelet account $270 \times 10^{4}$. Prothrombin time was 11 (range 9.3-12.3), INR 0.94, total serum bilirubin $0.79 \mathrm{mg} / \mathrm{dl}$. The B Child-Pugh classification passed to class A. AST-ALT were 59/56 UI/L respectively, and without encephalopathy. The level of portal hypertensive gastropathy observed in the endoscopic control was decreased to moderate.

\section{DISCUSSION}

The spleen is an important lymphoid organ, which has the function of storing blood, hematopoiesis, and immunizing power; however removal the spleen by laparoscopic or open procedures is an effective invasive treatment for spleen rupture, portal hypertension, hypersplenism, splenomegaly and idiopathic thrombocytopenic purpura. Even though, after splenectomy it can bring high risk of infection, immune function decline, and thrombocytosis., ${ }^{4,7}$ in this study they were not observed those complications. Generally, vaccines (pneumococcal vaccine, haemophilus influenza type b conjugate vaccine, and meningococcal conjugate vaccine and N. meningitidis) were administered 2 weeks before splenectomy to all patients. ${ }^{4,8-10}$ However all patients in our study had pneumococcal vaccine from 2 to 3 weeks before the splenectomy. The aim of present study was to evaluate relationships between degree of portal hypertension and follow-up, during six months after laparoscopic or open splenectomy with disconnection of short stomach or azygos veins.

The histological characteristics findings of PHG are dilated capillaries and venules in the mucosa and submucosa without erosion, inflammation, or fibrinous thrombi. ${ }^{11-13}$ Therefore, our patients were classified under the classification of portal hypertensive gastropathy according to new Italian Endoscopic Club and the commonly endoscopy classification: that's mean Mosaic like pattern: mild-diffusely pink areola, moderate-flat red spot in centre of pink areola, severe-diffusely red areola, red lesions of variable diameter, flat or slightly protruding, discrete o confluent. ${ }^{12}$ respectively, in the commonly endoscopy classification; F1, small straight varices; F2, 
The Role of Splenectomy with Vascular Disconnection in Pediatric Patients with Hypersplenism, Portal Hypertension and Liver Disease

enlarged tortuous varices that occupy less than one third of the lumen; and F3, large coil-shaped varices that occupy more than one-third of the lumen. ${ }^{3}$ Our patients had moderate to severe PHG that's mean F2 to F3 respectively.

Under physiologic condition, after splenectomy; there are proliferations of platelet megakaryocyte in bone marrow, induced platelets rapidly increased 2-6 times in the blood circulation. This effect was observed in our patient after the splenectomy. ${ }^{10,14}$ On the other hand, the risk of sepsis is greatest during the first 2 years after splenectomy. Children under 5 years old at the time of splenectomy have a greater risk for sepsis than older children and adult; therefore, in our study they had 2 patients $<5$ years without infections in the first 6 months after splenectomy. ${ }^{4,11,15}$ Pancreatic fistula is another rare complication in patient after laparoscopic splenectomy with prevalence range from $4.5 \%$ to $16 \%$ in patient with hematological diseases; Tsutsumi report that the incidence of pancreatic fistula after laparoscopic splenectomy in patient with hypersplenism due to liver cirrhosis is not low. However, we had not this complication in our patients. ${ }^{14}$

Hypertension gastropathy commonly encountered in patients with cirrhosis, with prevalence between $20 \%$ and $80 \%$ and observed more disease. In fact, hepatic venous pressure gradient (HVPG) is higher and systemic vascular resistance lower in patients with PHG. Prostaglandins, Nitric oxide synthase and local ischaemia also appear to play a role. ${ }^{3,12,16}$ Portal hypertension gastropathy is the most common gastric mucosal injury in patients with liver cirrhosis. Furthermore, there is an increase in total gastric blood flow but with a changed distribution: a relative decrease in blood flow to the mucosa and increased blood flow to the submucosa, muscle, and serosal layers. ${ }^{16}$ On the other hand, idiopathic non-cirrhotic intrahepatic portal hypertension is characterized by occlusion of the small intrahepatic portal vein radicles. ${ }^{17}$ Other disease that playing an important role in the portal hypertension is the portal cavernoma (PC) caused gastrointestinal bleeding among children, some studies mentioned at least one episode of upper gastrointestinal bleeding was present in approximately $80 \%$ of patients with PC or better known like cavernous transformation of the portal vein (CTP). ${ }^{7}$ In our review , fifty percent of patients had CTP with hypertensive gastropathy and gastrointestinal bleeding that is not different in literature report.

There are various proposal of treatment for PH; Kalambokis George N et al, in its brief report suggested that terlipressin should be considered the vasoconstrictor treatment of choice when cirrhotic patients with PH present with variceal bleeding or hepatorenal syndrome (HRS) in our series of four patients we used beta blockers and our patients had not HRS. ${ }^{9}$ In our study, Patients 1, 2 and 4 had more than two episodes of bleeding. The indications for splenectomy in cirrhotic patients are somewhat controversial. ${ }^{15}$ However, the most common indications of splenectomy are for hereditary spherocytosis, idiopathic thrombocytopenic purpura, sickle cell anemia and hematologic diseases; all of our patients had hypertensive gastropathy due to portal hypertension and other disease of the liver, we had one patient with sickle cell anemia that presented a good outcome after a laparoscopic splenectomy. ${ }^{11}$

Portal hypertension might eventually develop with esophagogastric varices, splenomegaly, and hypersplenism ${ }^{7}$ Like it was in our study, Guo H. reported presented nine cases with open retroperitoneal communicating branches (venous plexus of retzius), to prevent variceal bleeding in its study. However, in this same study they performed disconnection in the gastric fundus and lower esophagus in combination with splenectomy was performed in 36 patients with CTP and they observed thrombus formation in eight patients among them; surgical thrombus removal was performed in those patients. In fact, in our small series of cases we performed the disconnection in the mayor curvature (Coronary Venous and in lower esophagus) of the stomach with splenectomy and we had not thrombi in six months of following. For patients with cirrhosis, portal hypertensive bleeding and hypersplenism, open splenectomy with azygoportal disconnection is generally accepted as the most effective approach. However, one of the most serious perioperative complications during laparoscopic splenectomy disconnection (LSD) is rapid loss of large volumes of blood. In our series of cases, patients one and two had LSD without loss of large volumes of blood. ${ }^{8}$

American Research Journal of Pediatrics

Page 6 
The Role of Splenectomy with Vascular Disconnection in Pediatric Patients with Hypersplenism, Portal Hypertension and Liver Disease

The normal diameter of the portal vein ranges from 6 to $10 \mathrm{~mm}$; Li et al. demonstrated that portal vein diameter $>13 \mathrm{~mm}$ was an independent risk factor of portal thrombosis after open splenectomy in cirrhotic patients with hypersplenism. ${ }^{1}$ However, some authors reported systemic inflammatory response syndrome experienced in $54 \%$ and the portal vein thrombosis experienced in $17 \%$ of the patients. In this study one patient had systemic inflammatory with good answer to the treatment. ${ }^{15}$ Portal vein thrombosis is a potentially lethal complication after splenectomy with reported incidence of $6 \%$ in children and more than $55 \%$ in adults. ${ }^{1,4,11}$ The mechanism and risk factors of this complication are poorly understood, especially in cirrhotic patients with portal hypertension. ${ }^{1}$ Fortunately, in our four pediatric patients, this complication was not observed.

Severe complicationssuchasuppergastrointestinalbleeding,refractoryascites, jaundice, hepaticencephalopathy, bowel edema acute ischemic necrosis, ileus and other serious complications were not observed also in this study. Some adult studies have demonstrated with splenectomy, portal vein pressure is reduced, which alleviates esophagogastric varices and prevents esophagogastric variceal bleeding. Furthermore, several importantaspects of chronic liver diseases still remain to be fully studied. Splenectomy improves also liver function. Similarly situations, were observed in our four pediatric patients. ${ }^{13,7,11}$ Bai et al. reported that, azygoportal disconnection can effectively reduce the recurrence rate of esophagogastric variceal bleeding, which is a major cause of death in patients with portal hypertension due to cirrhosis in adult population similarly effects were observed in our series of pediatric patients. ${ }^{5}$ Finally, Yamamoto et al. observed that the liver function had changed to childPugh class C after progression of liver disease with portal vein invasion at 1 year after splenectomy. In our four patients with portal hypertension we observed diminution of portal hypertension and Child-Pugh class at 6 months of the procedure was maintained or change to a better score. ${ }^{5,7}$ The mechanism underlying this functional amelioration of the liver after splenectomy has yet to be fully elucidated. ${ }^{6}$ Furthermore, plateletderived serotonin has recently been shown to be important in liver regeneration.

\section{CoNCLUSION}

The splenectomized pediatric patient requires careful monitoring in the early postoperative period to identify some complications like formation of portal thrombi and infections; in this study we observed a good outcome on the platelets account without bleeding, and amelioration of the portal hypertension gastropathy similarly a better function of liver or prolongation of the time of liver deterioration. Through this time of follow-up any patient required variceal treatment by endoscopic method and the risk of variceal bleeding was decreased. The weakness of this study was the number of patients and carefully patient selection.

\section{REFERENCES}

1. Jiang Guo-qing, Bai Dou-sheng, Chen Ping, et al. Risk Factor for Portal Vein System Thrombosis After Laparoscopic Splenectomy in Cirrhotic Patients with Hypersplenism. Journal of Laparoendoscopic \& Advance Surgical Techniques. 2016; 26(6): 419-23.

2. Kawanaka H, Akahoshi T, Kinjo N, et al. Effect of laparoscopic splenectomy on portal haemodynamics in patients with liver cirrhosis and portal hypertension. BJS. 2014; 101: 1585-93.

3. Silkauskaité Vilma, Pranculis Andrius, Mitraité Dalia, et al. Hepatic venous gradient measurement in patient with liver cirrhosis: a correlation with disease severity and variceal bleeding. Medicina (kaunas). 2009; 45(1): 8-13.

4. Buzelé R, Barbier L, Sauvanet A, et al. Medical complications following splenectomy. Journal of visceral Surgery. 2016; 153: 277-86.

5. Bai Dou-sheng, Qian Jian-Jun, Chen Ping, et al. Laparoscopic azygoportal disconnection with and without splenectomy for portal hypertension. International Journal of Surgery. 2016; 34: 116-21. 
The Role of Splenectomy with Vascular Disconnection in Pediatric Patients with Hypersplenism, Portal Hypertension and Liver Disease

6. Yamamoto Naoki, Okano Keiichi, Oshima Minoru, et al. Laparoscopic splenectomy for patients with liver cirrhosis: Improvement of liver function in patients with Child-Pugh class B. Surgery. 2015; 6:1538-44.

7. Guo Honjie, Hao Fabao, Guo Chunbao, et al. Outcome of the pediatric patient with portal cavernoma: The retrospective study for 10 years focusing on recurrent variceal bleeding. Gastroenterology Research and Practice. 2016:1-8.

8. Jiang Guo-Qing, Bai Dou-Sheng, Chen Ping, et al. Modified laparoscopic splenectomy and azygoportal disconnection combined with cell salvage is feasible and might reduce the need for blood transfusion. World Journal of gastroenterology. 2014; 20 (48): 18420-26.

9. Kalambokis George N, Pappas Konstantinos, Tsianos Epameinondas V. terlipressin improves pulmonary pressures in cirrhotic patients with pulmonary hypertension and variceal bleeding or hepatorenal syndrome. Hepatobiliary Pancreat Dis Int. 2012; 11(4): 434-37.

10. Qian Yu-Yian, Li Kun. The early prevention and treatment of PVST after laparoscopic splenectomy: A prospective cohort study of 130 patients. International Journal of Surgery. 2017; 44: 147-51.

11. Luoto Topi T, Pakarinen Mikko P, Koivusalo Antti, et al. Long-term outcomes after pediatric splenectomy. J. Surgery. 2016; 159(6):1583-90.

12. Patwardhan V: R, Cardenas A. Review article: the management of portal hypertensive gastropathy and gastric antral vascular ectasia in cirrhosis. Aliment Pharmacol Ther. 2014; 40: 354-62.

13. Su An-ping, Zhang Zhao-Da, Tian Bo-Le. Trnasjugular inthrahepatic portosystemic shunt versus open splenectomy and esophagogastric devascularization for portal hypertension with recurrent variceal bleeding. Hepatobiliary Pancreat Dis Int. 2017; 16(2):169-75.

14. Tsutsumi Norifumi, Tomikawa Morimasa, Akahoshi Tomohiko, et al. Pancreatic fistula after laparoscopic splenectomy in patients with hypersplenism due to liver cirrhosis: effect of fibrin glue and polyglycolic acid felt on prophylaxis of post operative complications. The American Journal of Surgery. 2016; 212(5): 882-88.

15. Du Zhaoqing, Dong Jian, Zhang Jia. Incidence and risk factors associated with a high comprehensive complication index score after splenectomy in cirrhotic patients with hypersplenism. Journal of Surgical research. 2018; 222: 69-74.

16. Kumar Ashish, Mishra Smurti Ranjan, Sharma Praveen. Clinical, Laboratory, and Hemodynamic Parameters in Portal Hypertensive gastropathy. A study of 254 cirrhotics. J Clin gastroenterol. 2010; 44 (4): 294-00.

17. Goel Ashish, Raghupathy V, Amirtharaj G.J, et al. AAMTS13 missense variants associated with defective activity and secretion of ADAMTS13 in a patient with non-cirrhotic portal hypertension. Indian J Gastroenterol. 2017; 36(5): 380-89.

Citation: Pierre Jean Aurelus, Hermilo De La Cruz Yáñez, Edwin Allen Mejía Solís, Elvia Ximena Lemus Manjarrez, María Antonieta Julián Núñez, Rafael Roberto Zapata Carrión. “The Role of Splenectomy with Vascular Disconnection in Pediatric Patients with Hypersplenism, Portal Hypertension and Liver Disease". American Research Journal of Pediatrics. 2018; 2(1): 1-8.

Copyright (C) 2018 Pierre Jean Aurelus, Hermilo De La Cruz Yáñez, Edwin Allen Mejía Solís, Elvia Ximena Lemus Manjarrez, María Antonieta Julián Núñez, Rafael Roberto Zapata Carrión. This is an open access article distributed under the Creative Commons Attribution License, which permits unrestricted use, distribution, and reproduction in any medium, provided the original work is properly cited. 